\title{
Is Doubling of Serum Creatinine a Valid Clinical 'Hard' Endpoint in Clinical Nephrology Trials?
}

\author{
H.J. Lambers Heerspink ${ }^{a} \quad$ V. Perkovic ${ }^{b} \quad$ D. de Zeeuw ${ }^{a}$ \\ a Department of Clinical Pharmacology, University Medical Center Groningen, University of Groningen, \\ Groningen, The Netherlands; ${ }^{b}$ The George Institute for International Health, University of Sydney, \\ Sydney, N.S.W., Australia
}

\section{Key Words}

Randomized controlled trial $\cdot$ Doubling of serum

creatinine $\cdot$ Nephrology

\begin{abstract}
The composite of end stage renal disease (ESRD), doubling of serum creatinine and (renal) death, is a frequently used endpoint in randomized clinical trials in nephrology. Doubling of serum creatinine is a well-accepted part of this endpoint because a doubling of serum creatinine reflects a large sustained change in glomerular filtration rate (GFR) and predicts the development of ESRD. Although doubling of serum creatinine is frequently used, the validity of using this outcome as part of a composite endpoint is hampered by various factors. Firstly, serum creatinine may reflect changes in muscle mass unrelated to true GFR changes. Secondly, changes in serum creatinine may reflect hemodynamic changes in renal perfusion and not a structural effect on renal function. Finally, doubling of serum creatinine is an arbitrary choice and different proportional changes may represent a better indicator for ESRD. In this minireview, each of these factors will be discussed and recommendations are made for interpretation of clinical trials using doubling of serum creatinine as a composite endpoint in nephrology trials.

Copyright $\odot 2011$ S. Karger AG, Basel
\end{abstract}

The final goal of a drug development plan is to determine the efficacy and safety of a new intervention. Studies should use meaningful clinical endpoints to most reliably assess the clinical impact and tolerability of a new intervention, particularly those that reflect how a patient feels, functions and how long a patient survives. However, in practice, clinical-endpoint studies are not always achievable. Some clinical endpoints occur rarely while others may take a long time to develop. Composite endpoints are often used to help address this issue by combining more than one event of interest. As a consequence, the event rates in trials with composite endpoint are higher so that fewer participants are required, and/or the duration of the trial is shortened.

In nephrology trials, the composite of end stage renal disease (ESRD), defined as the need for chronic dialysis or renal transplantation, doubling of serum creatinine from baseline and death, is a commonly used composite endpoint and has been used in various clinical trials in nephrology [1-3]. The doubling of the serum creatinine component is a well-accepted part of the composite because large long-term changes in serum creatinine are thought to reflect a structural renal function decline [4]. Since glomerular filtration rate (GFR) generally decreases linearly over time in individual patients, a doubling of serum creatinine should reflect a sustained reduction in

\section{KARGER}

Fax +41613061234 E-Mail karger@karger.ch www.karger.com
Hiddo J. Lambers Heerspink, PharmD, PhD

Department of Clinical Pharmacology, University Medical Center Groningen

University of Groningen, Ant Deusinglaan 1

NL-9713 AV Groningen (The Netherlands)

Tel. +31 50363 2810, E-Mail H.j.lambers.heerspink@ med.umcg.nl 
GFR, and be an important step on the path towards ESRD.

Although doubling of serum creatinine is frequently used, the validity of using this outcome (or its equivalent changes in serum creatinine or GFR) as part of a composite endpoint is hampered by various factors: (a) creatinine levels are highly dependent on muscle mass, so that changes in GFR derived from serum creatinine levels may represent highly variable changes in kidney function at different creatinine levels; (b) serum creatinine changes do not necessarily reflect true changes in GFR, but may instead reflect a hemodynamic change in renal perfusion (and not a structural effect on renal function), and (c) doubling of serum creatinine is an arbitrary choice and different proportional changes may represent a better indicator for a hard renal outcome (ESRD).

\section{Serum Creatinine Changes Not due to True GFR Change}

Changes in serum creatinine can be attributed not only to renal structural changes, and thus changes in GFR, but serum creatinine may also change as a consequence of changes in muscle mass, food intake, concomitant medication and laboratory conditions [5]. Firstly, creatinine production from creatine and phosphocreatine occurs exclusively in muscles. Serum creatinine levels are therefore proportional to muscle mass. Hence, changes in muscle mass over time as a consequence of aging rather than GFR may influence creatinine levels. Another source of variability in serum creatinine levels is changes in diet. Creatine from ingested meat can account for up to $30 \%$ of total creatinine excretion and thus, variability in meat intake over time can be another contributor to variability in serum creatinine levels. In addition, serum creatinine levels also vary according to the source of proteins in the diet. Proteins from red meat diet appear to exert direct (hemodynamic) effects on GFR while vegetable proteins seem to have a lesser effect. Thirdly, it is well recognized that creatinine is partly secreted by the renal tubule. Changes in tubular secretion of creatinine account for another source of variability in serum creatinine [6]. This is particularly relevant in patients with overt proteinuria and hypoalbuminemia in whom tubular secretion of creatinine is markedly increased. If tubular secretion of creatinine was constant, a consistent correction factor could be introduced to obtain a more accurate estimate of GFR. However, studies have shown that within an individual, secretion of cre- atinine may fluctuate over time, which makes the introduction of such a correction factor impossible [6]. In addition, tubular secretion can be blocked by various frequently used drugs, including cimitidine, trimethoprim and pyrimethamine. The concomitant use of these drugs may create another source of variability in serum creatinine levels. Finally, the measurement of serum creatinine itself is prone to large variability. The alkaline picrate (Jaffé) method by Bonsnes and Taussky [7] has been used by many laboratories to measure serum creatinine. However, this assay is subject to interference from other chromogens, causing an overestimation of serum creatinine by as much as $15-25 \%$. More recent enzymatic assays do not suffer from this problem. However, despite the use of these novel assays, large variability in serum creatinine measurements across laboratories is still reported [8]. This variability renders estimating GFR equations less accurate and precise. Calibration against an isotope dilution mass spectrometry (IDMS) standard mitigates part of the laboratory variability [9]. Hence, serum creatinine may vary over time due to various factors not relating to kidney function. Failure to consider these sources of variability in serum creatinine may lead to misinterpretation of the long-term effects of interventions on the doubling of the serum creatinine endpoint.

\section{Changes in Serum Creatinine Reflect a Hemodynamic GFR Change}

Creatinine excretion, and therefore serum levels, is highly dependent on renal perfusion. Any condition that reduces renal perfusion may reversibly increase serum creatinine levels without necessarily impacting the kidney structurally in any way. The classic example of this is volume depletion, where restoration of plasma volume leads to prompt and complete normalization of serum creatinine levels.

Drugs and dietary interventions may also affect serum creatinine differently over time: initially a drug may increase serum creatinine via hemodynamic effects (reduced glomerular perfusion being reflected in a fall in GFR) whereas the long-term effects may go in the opposite direction (renoprotection) as a result of renal structural effects. This complicates the interpretation of the doubling of serum creatinine endpoint. The doubling could either be the consequence of the initial (reversible) hemodynamic effect or the result of a structural worsening of renal function. A classic example of this 
situation is when angiotensin-converting enzyme inhibitors or angiotensin receptor blockers are used. Despite demonstrated long-term benefits on kidney function, acute increases in creatinine that reflect hemodynamic changes are commonly seen when treatment is commenced.

In clinical care, patients are treated to delay or prevent the structural worsening of renal function. Hence, when determining the renoprotective effects of an intervention, one needs to ascertain that the effect of the intervention delays the long-term structural renal function decline and is not solely caused by an acute, reversible hemodynamic effect. The difficulty in differentiating between this hemodynamic and long-term structural change is illustrated by the recent ACCOMPLISH renal substudy [10]. Bakris et al. [10] reported beneficial effects of amlodipine compared to hydrochlorothiazide when used on a background of angiotensin-converting enzyme inhibition for renal function. However, in the ACCOMPLISH trial, the observed differences in kidney disease endpoints were solely observed for doubling of serum creatinine while the most relevant ESRD endpoint rarely occurred. The possibility thus exists that the endpoint in ACCOMPLISH was driven by a hemodynamic effect rather than an effect on structural renal function. This possibility is substantiated by previous studies demonstrating that diuretics cause a distinct acute fall in GFR (increase in serum creatinine) at onset of therapy while calcium channel blockers increase GFR (decrease serum creatinine) during the first month of therapy $[11,12]$. Slagman et al. [13] recently analyzed data from a randomized controlled trial and confirmed that withdrawal of hydrochlorothiazide resulted in a marked rise in creatinine clearance that was paralleled by a distinct fall at reinstitution. These data highlight that initiation of hydrochlorothiazide causes a hemodynamic fall which is reversible after stopping therapy.

Do we have evidence that such a scenario has occurred in the ACCOMPLISH trial as well? The ACCOMPLISH study group presented data at the World Congress of Nephrology 2009 with respect to the estimated GFR (eGFR) changes during the ACCOMPLISH trial [14]. During the first 3 months of the trial, the mean eGFR showed a distinct fall in the hydrochlorothiazide group while eGFR rose in the amlodipine group. The subsequent eGFR slope from month 3 until month 36 was similar between the two treatments, suggesting that any differences in the number of doubling of serum creatinine endpoints may be attributed to the initial hemodynamic fall in eGFR. The month-36 eGFR level was higher in patients assigned to amlodipine and thus one may argue that amlodipine confers additional renoprotection. However, as shown by Slagman et al. [13], stopping the drugs will likely result in an eGFR change in opposite direction as the initial effect and thus the net eGFR change may be similar between hydrochlorothiazide and amlodipine. Unfortunately, serum creatinine was not measured after termination of the ACCOMPLISH trial, which makes it impossible to verify this possibility. Nevertheless, the ACCOMPLISH trial highlights the caution that is needed when interpreting the effect of a drug on the doubling of serum creatinine endpoint if the data are not substantiated by hard renal endpoint data. Failure to take into account the acute reversible hemodynamic drug effect may lead to misinterpretation of the true long-term effects. We therefore recommend that any trial using slope outcomes defined by GFR use a baseline measurement some time after randomization to take account of the hemodynamic drug effect.

\section{Is Doubling of Serum Creatinine the Right Measure for Progression to ESRD?}

The first trial that included the doubling of serum creatinine as part of the composite renal endpoint was the Captopril Collaborative Study Group trial [15]. The choice of a doubling in serum creatinine as part of the composite endpoint was based on logic reasoning in that patients with a halving of kidney function within a defined period of time will likely proceed to dialysis or renal transplantation. The doubling was, however, not substantiated by scientific evidence demonstrating that a doubling in serum creatinine confers the most accurate increase for establishing true renal function decline. It may be possible that smaller increases in serum creatinine convey a more accurate change to predict the clinical endpoint ESRD. If so, it would facilitate clinical drug trials as the duration of clinical drug trials could be shortened and fewer patients would be required to achieve similar statistical power. Conversely, the acute hemodynamic effects many interventions exert may have a relatively bigger impact if a smaller increase in serum creatinine is used as the composite of a clinical endpoint. This is exemplified in figure 1 . Thus, the proportionally larger impact of the hemodynamic renal effects of many drugs will likely counterbalance the potential benefits of using smaller increases in serum creatinine as endpoint in clinical drug trials. Another point of consideration is that the absolute GFR decline to achieve a doubling in serum cre- 


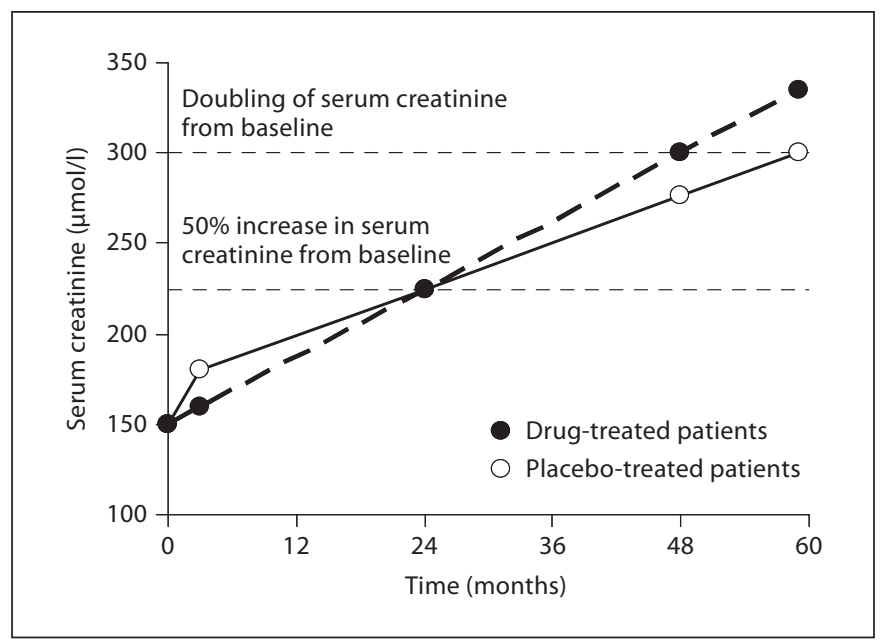

Fig. 1. An example assuming a drug causes an acute rise in serum creatinine of $20 \%$ during the first 3 months of therapy with a subsequent rise of $25 \%$ during the following 21 months. Placebotreated patients progressively show an increase in serum creatinine of $50 \%$ during the first 2 years. If the clinical endpoint is defined as an increase in serum creatinine of $50 \%$, both treatment groups would achieve the endpoint after 2 years. However, the endpoint in the active treatment arm is largely based on the initial hemodynamic effect of the drug. In other words, the rate of longterm structural renal function decline from month 3 onwards is lower in patients treated with the new drug but is negated by the initial hemodynamic effect. Hence, if a $50 \%$ increase in serum creatinine is used as the endpoint, the renoprotective effects of the drug would not be recorded. In contrast, if a doubling of serum creatinine is used as the clinical endpoint, the follow-up period is sufficiently long to observe the beneficial effect of the drug: patients treated with the drug would reach the endpoint after 59 months, whereas placebo-treated patients reach the endpoint after 48 months.

atinine diminishes at higher serum creatinine because of

\section{Conclusion}

The ideal hard endpoint for nephrology trials is the occurrence of ESRD or renal death. Where financial or practical constraints render it impossible to use ESRD or renal death alone as the primary endpoint, doubling of serum creatinine can be considered as an additional component of the composite endpoint. This may be particularly appropriate where the intervention does not have a direct effect on serum creatinine levels. However, if doubling of serum creatinine is to be used, changes in serum creatinine should be carefully analyzed and reported. The variable acute and chronic effects of many drugs on renal function suggest caution should be used when interpreting clinical drug trials using doubling of serum creatinine or the rate of GFR decline as outcome. Therefore, for appropriate interpretation of clinical drug trials recording only doubling of serum creatinine and/or GFR changes, two criteria should be fulfilled: Firstly, serum creatinine should be measured after discontinuation of the study medication to establish whether acute changes during the initial months of therapy are reversible. Secondly, the short-term and long-term effects on serum creatinine and GFR should be separately analyzed and reported to allow correct interpretation of the effect of a drug on renal function. Further studies are urgently required to answer the question whether a doubling or another magnitude of change in serum creatinine is the best composite of a renal endpoint. the reciprocal relationship between serum creatinine and GFR. Indeed, in the captopril trial, most doubling of serum creatinine endpoints were observed in patients with higher entry serum creatinine levels, between 1.5 and 2.0 $\mathrm{mg} / \mathrm{dl}[14]$. This indicates that the sensitivity to detect absolute changes in GFR is higher as serum creatinine rises. Thus, doubling of serum creatinine may be an appropriate endpoint in trials recruiting patients with high serum creatinine levels (e.g. $>1.5 \mathrm{mg} / \mathrm{dl}$ ) but may be less useful in trials when the serum creatinine entry levels are lower (e.g. $<1.0 \mathrm{mg} / \mathrm{dl}$ ) Appropriate designed analyses of randomized controlled trials are needed to establish which proportional increase in serum creatinine can be reliably used as composite of a clinical endpoint before these can be accepted routinely.

References
-1 Brenner BM, Cooper ME, de Zeeuw D, Keane WF, Mitch WE, Parving HH, et al: Effects of losartan on renal and cardiovascular outcomes in patients with type 2 diabetes and nephropathy. N Engl J Med 2001;345: 861-869.

-2 Lewis EJ, Hunsicker LG, Clarke WR, Berl T, Pohl MA, Lewis JB, et al: Renoprotective effect of the angiotensin-receptor antagonist irbesartan in patients with nephropathy due to type 2 diabetes. N Engl J Med 2001;345: 851-860.

3 Lambers Heerspink HJ, Fowler MJ, Volgi J, Reutens AT, Klein I, Herskovits TA, et al: Rationale for and study design of the sulodexide trials in type 2 diabetic, hypertensive patients with microalbuminuria or overt nephropathy. Diabet Med 2007;24:1290-1295.

4 Products TEAftEoM: Note for guidance on clinical investigations of medicinal products in the treatment of diabetes mellitus, 2002

Lambers Heerspink/Perkovic/de Zeeuw 
5 Fuller NJ, Elia M: Factors influencing the production of creatinine: implications for the determination and interpretation of urinary creatinine and creatine in man. Clin Chim Acta 1988;175:199-210.

6 Levey AS, Berg RL, Gassman JJ, Hall PM, Walker WG: Creatinine filtration, secretion and excretion during progressive renal disease. Modification of Diet in Renal Disease (MDRD) Study Group. Kidney Int Suppl 1989;27:S73-S80.

7 Bonsnes RW, Taussky HH: On the colorimetric determination of creatinine by the Jaffé reaction. J Biol Chem 1945;158:581-591.

-8 Joffe M, Hsu CY, Feldman HI, Weir M, Landis JR, Hamm LL: Variability of creatinine measurements in clinical laboratories: results from the CRIC study. Am J Nephrol 2010;31:426-434. $\checkmark 9$ Levey AS, Coresh J, Greene T, Stevens LA, Zhang YL, Hendriksen S, et al: Using standardized serum creatinine values in the modification of diet in renal disease study equation for estimating glomerular filtration rate. Ann Intern Med 2006;145:247254.

${ }_{10}$ Bakris GL, Sarafidis PA, Weir MR, Dahlof B, Pitt B, Jamerson K, et al: Renal outcomes with different fixed-dose combination therapies in high-risk hypertensive patients. Lancet 2010;375:1173-1181.

11 Vogt L, Waanders F, Boomsma F, de Zeeuw D, Navis G: Effects of dietary sodium and hydrochlorothiazide on the antiproteinuric efficacy of losartan. J Am Soc Nephrol 2008; 19:999-1007.

12 Herlitz H, Harris K, Risler T, Boner G, Bernheim J, Chanard J, Aurell M: The effects of an ACE inhibitor and a calcium antagonist on the progression of renal disease: the Nephros Study. Nephrol Dial Transplant 2001; 16:2158-2165.
13 Slagman MC, Navis G, Laverman GD: Reversible effects of diuretics added to reninangiotensin-aldosterone system blockade: impact on interpretation of long-term kidney function outcome. Am J Kidney Dis 2010;56:601-602.

14 Sarafidis PA, Bakris G, Weber M, Pitt B, Dahlof B, Velazquez EJ, et al: Changes in glomerular filtration rate with benazepril plus amlodipine or benazepril plus hydrochlorothiazide treatment in hypertensive patients at high cardiovascular risk; an analysis of the ACCOMPLISH trial. http://wwwabstracts 2viewcom/wcn/viewphp?nu=WCN09L_455 2009 (30 November, 2010).

15 Lewis EJ, Hunsicker LG, Bain RP, Rohde RD: The effect of angiotensin-converting-enzyme inhibition on diabetic nephropathy. The Collaborative Study Group. N Engl J Med 1993;329:1456-1462.

\section{Editorial Comment}

Meguid El-Nahas, Sheffield

The minireview by Lambers Heerspink, Perkovic and de Zeeuw addresses the important issue of endpoints in clinical trials on progressive chronic kidney disease (CKD). It examines in detail the value of the commonly used endpoint for progressive CKD, namely the doubling of serum creatinine. It reminds the readers of the limitations of such a measurement, as all too often the variability of serum creatinine measurements as well as confounders are overlooked. Changes in serum creatinine estimation in CKD can be affected by a number of factors including creatine/creatinine intake, metabolism, filtration and secretion. Often changes in serum creatinine are solely equated with GFR! I remember all too well the plethora of clinical trials in the 1970s and 1980s that relied on changes/fall in serum creatinine levels to argue for a beneficial effect of low-protein diets on the progression of CKD, overlooking the impact of such diets of creatinine intake, metabolism and muscle mass. Unfortunately, these lessons have not been learned as serum creati- nine and derived GFR calculations continue to be used in large clinical trials to argue for a beneficial effect on GFR! There is little doubt that clinical trials should rely on measured GFR (mGFR) to estimate the impact on progression of CKD. This should include changes in mGFR over time along with the slope of mGFR/time; the former alone can be misleading if baseline and final mGFR are solely relied upon, thus the need for a negative mGFR slope over the observation time based on a number of measurements to confirm the nature of the observed trend in renal function. Nephrologists have been criticized over the years for the quality of the clinical trials undertaken - all too often underpowered with inadequate sample size. This has been largely addressed over the last decade with larger trials. Nowadays, we could still be criticized for using inappropriate single or composite endpoints including serum creatinine measurements or derived eGFRs! 